\title{
Emergency Airway Obstruction in Newborn Due to Congenital Saccular Cyst
}

\author{
Itzhak Braverman $^{1}$, Galit Avior ${ }^{1}$, Michael Feldman², Andrei Gubarev ${ }^{1}$ Ronnie Stein², \\ Hakeem Abu Ras ${ }^{3}$, Abdel-Rauf Zeina ${ }^{4}$ \\ ${ }^{1}$ Otolaryngology_-Head and Neck Surgery Unit, The Hillel Yaffe Medical Center, Hadera, Israel \\ ${ }^{2}$ Newborn and Neonatal Care Department, The Hillel Yaffe Medical Center, Hadera, Israel \\ ${ }^{3}$ Department of Anesthesiology, The Hillel Yaffe Medical Center, Hadera, Israel \\ ${ }^{4}$ Department of Radiology, The Hillel Yaffe Medical Center, Hadera, Israel \\ Email: braverman@hy.health.gov.il
}

Received October 1, 2012; received November 18, 2012; accepted November 29, 2012

\begin{abstract}
Laryngeal cyst causing neonatal airway obstruction during labor is a very rare condition [1]. Congenital laryngeal cysts are a rare cause of neonatal airway obstruction. Traditionally, these cysts have been treated surgically by endoscopic excision or marsupialization. However, the cyst often extends beyond the larynx. We describe a case of a newborn that, during delivery, became cyanotic due to airway obstruction and respiratory distress. To the best of our knowledge this is the first report of a saccular cyst obstructing airway during birth prior to intubation. The immediate and late treatments together with a literature review are described.
\end{abstract}

Keywords: Sacullar Cyst; Airway Obstruction; Obstructive Sleep Apnea; Congenital Laryngeal Cysts

\section{Case Report}

A newborn girl was delivered by caesarian section at age 33 weeks and developed marked respiratory distress. She became cyanotic with airway obstruction in the operating room, immediately after birth. During intubation laryngoscopy, performed by a senior anesthetist, a large hypopharyngeal obstructing mass was noted in the supraglottic region. In order to save the infant, the otolaryngologist and the senior anesthesiologist decided to immediately aspirate the fluid from the mass that looked cystic. A dark cystic fluid was extracted and the larynx became visible. Intubation was performed to keep the airway open. The newborn was evaluated by endoscopic fiberoptic laryngoscopy (Figure 1), which showed a cystic supraglottic laryngeal mass that was a large laryngeal cyst compressing the airway inlet and the laryngeal tube, originating from the right side. The cyst looked smooth, occupying the aryepiglottic fold. MRI was performed (Figure 2), which showed a cystic lesion consistent with right lateral saccular laryngeal cyst as well as the airway displacement. A diagnosis of saccular laryngeal cyst was made and the newborn was scheduled for endoscopic removal of the cyst.

\section{Endoscopic Removal of Congenital Saccular Cyst}

At age 19 days, under general anesthesia, using a Storz- pediatric laryngoscope and a Storz 0 degree $4 \mathrm{~mm}$ rigid endoscope with high definition monitor, the saccular cyst was viewed and dissected using Starion forceps (Figure 3). This technology uses heat to seal and divide soft tis-

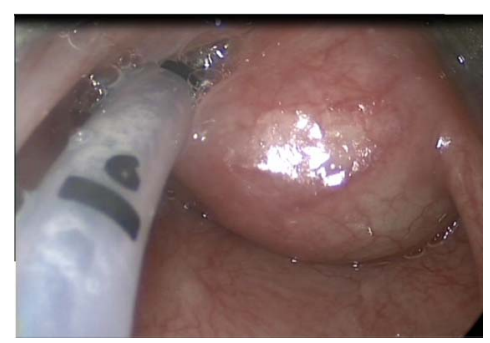

Figure 1. It shows a cystic supraglottic laryngeal mass.

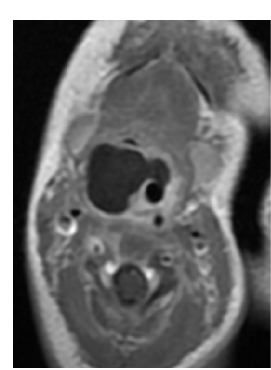

(a)

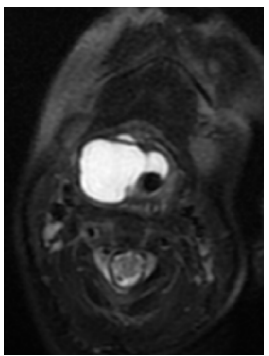

(b)
Figure 2 I. Axial T1-weighted (a) and T2-weighted (b) MRI images of the neck demonstrating a well defined cystic lesion consistent with right lateral saccular laryngeal cyst. 


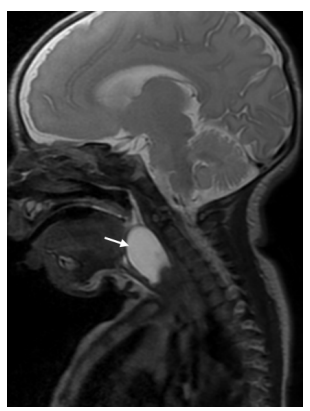

(a)

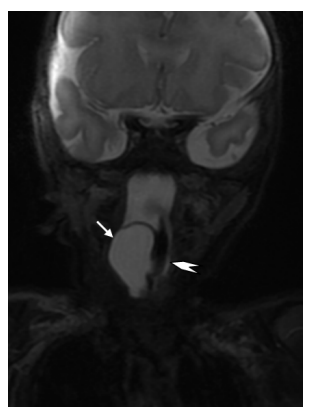

(b)
Figure 2 II. Sagittal (a) and coronal (b) T2-weighted MRI images of the neck showing the size and the extent of the saccular laryngeal cyst (arrows) as well as the airway displacement (arrowhead).

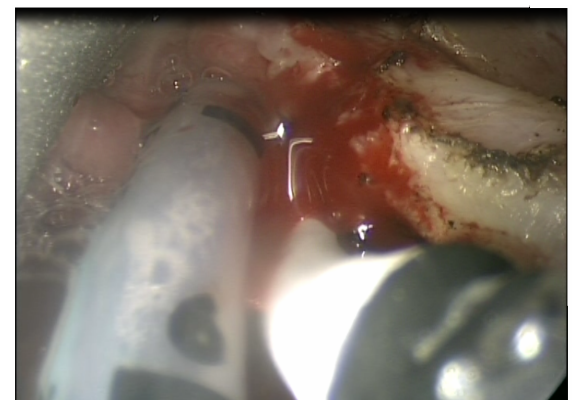

Figure 3. Intra-operative endoscopic removal of the saccular cyst that was viewed and dissected using Starion forceps.

sues by breaking protein bonds. Heating and cooling protein-based structures causes the molecules to unravel and then form a coagulum. By employing a proprietary temperature and pressure profile to modify proteins, Starion's instruments create a high-integrity seal and a clean divi sion. We wanted no electrical current to pass through the body during this process. The resistive heating element is powered by low voltage direct current. A graded thermal profile is created with a narrow higher temperature zone for cutting and a lower temperature-coagulating zone on each side, allowing the device to simultaneously seal and divide.

With this temperature gradient, we were able to both seal and divide tissue in a way that minimizes damage to surrounding tissue.

As we worked very near the larynx of this small infant, we did not want to use high temperature devices. The cyst was removed by marsupialization and sent to the histological laboratory (Figure 4).

The infant was treated with steroids and antibiotics for one week and extubation was performed. There was no airway problem and $\mathrm{O}_{2}$ saturation was kept at a high level.

The patient was discharged and asked to return for follow-up in the ENT clinic.
Follow-up after 16 months revealed a normally developing infant with no voice problems and no difficulties in eating or breathing (Figure 5).

\section{Discussion}

Congenital laryngeal cysts are thought to arise from obstruction of saccular ducts or from atresia of the saccule itself $[2,3]$. Its anatomic location allows a laryngeal cyst to expand through various structurally weaker areas of the larynx. Lateral extension through the thyroid membrane has been reported. These cysts can also expand in an inferior direction, steered by the conus elasticus, causing splaying and thinning of the lateral thyroid cartilage, prolapsing through the cricothyroid membrane, and extending inferiorly into the paratracheal region.

Saccular cysts are part of laryngeal cysts presenting with variable degrees of airway obstruction, hoarseness, and dysphagia.

The first classification of cystic laryngeal lesions was described by Desanto et al. [4]. The authors divided all laryngeal cysts into saccular, ductal, and thyroid cartilage foraminal cysts. However, they did not include congenital cysts as a separate entity and did not aim to guide the surgical management of these lesions.

In 1997, Arens and colleagues [5] created a new classification system in which the location of the cyst and histomorphology were taken into consideration. In this classification system, laryngeal cysts were classified as congenital, retention, or inclusion cysts.

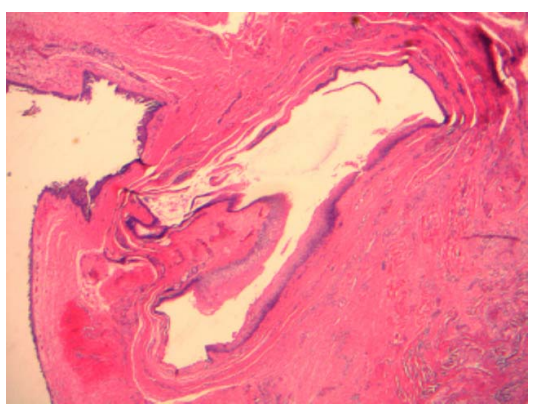

Figure 4. Shows the histological finding of the cyst.

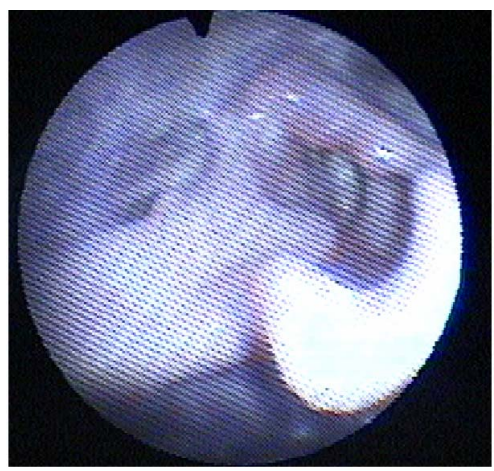

Figure 5. Shows the larynx following the operation. 
Vito Forte from Toronto [1] introduced a new classification system for congenital laryngeal cysts. Type I: Intralaryngeal cysts remain within confines of the larynx (endodermal elements only). Type II: Extralaryngeal extension cysts extend beyond the confines of the larynx. IIa: Endodermal elements only. IIb: Endodermal andmesodermal elements (laryngotracheal duplication or diverticulum).

Classically, congenital laryngeal cysts have been histologically shown to be comprised of squamous or respiratory epithelium with a fibrous stroma (i.e., endodermal derivatives) [4].

The saccule is a membranous pouch located between the ventricular fold and the inner surface of the thyroid cartilage. The normal mucous membrane surface of the saccule is covered with openings of 60 - 70 mucous glands [5].

The pathophysiology of saccular cysts results from obstruction of the laryngeal saccule orifice in the ventricle, with resultant mucus retention in the saccule [1]. Endoscopy is the gold standard for diagnosis of saccular cysts. Endoscopic evaluation reveals a cystic lesion containing thick, mucoid fluid emanating from behind the aryepiglottic fold in the case of lateral cysts, or from the ventricles, and protruding into the laryngeal lumen in the case of anterior cysts [6]. Needle aspiration may be useful in diagnosing the lesion, but drainage of the cyst offers only temporary treatment [6].

Marsupialization may be adequate for the treatment of small saccular cysts. However, in the case of recurrence or large cysts, endoscopic or open excision of the cyst is required so as to remove the cystic tissue completely [6].

Preoperative imaging with CT or MRI is an integral part of this new classification system. Every patient should have adequate imaging before any surgical intervention. It is essential in determining the size, location, and anatomic relations of a cyst, with a view toward selecting the optimal modality of surgical management [1].

Open surgical approaches for congenital laryngeal cysts have been described $[1,8]$ and some authors believe that the best treatment in terms of healing is complete excision of the cyst. For this reason, some authors recommend a cervical approach in the first intention, especially in case of large cysts, since it offers good extramucosal exposure of the paralaryngeal space, facilitating cyst excision with minimal morbidity [8].

The technique used in all Forte $\mathrm{V}$ cases was an anterior approach midline thyroidotomy. The authors recommend following the cyst to its intralaryngeal origin.

The midline thyroidotomy technique has, in their experience, proven to be an effective, safe way to completely remove congenital laryngeal cysts.

This new classification system is based on the anatomic extension of the cyst and histopathology. This new system aims to aid the surgeon with surgical management of these cysts, with the goal of avoiding unnecessary open surgical procedures and also, to preventing recurrence of the cyst and the need for repeated endoscopic treatments or tracheotomy.

Airway-obstructing saccular cysts in adults are rare laryngeal anomalies. Treatment with tracheotomy may be needed for control of the airway. We did not want to perform a tracheostomy in our neonate, although this possibility was an option.

Our case demonstrates that large saccular cysts, defined as Type II, can be removed endoscopically with less morbidity employing the new cutting tool technology, with less thermal injury and very good visualization using endoscopy and a high definition monitor.

It should be remembered that if endoscopic excision of the cyst lining is incomplete, secondary scarring and fibrosis at the surgical site may cause expansion of the cyst in a direction away from the fibrosis, along a path of least resistance.

To ensure that simple marsupialization is successful, one must create a wide opening in the cyst wall to allow continued decompression. The location of the cyst opening, the laxity of the tissues at the orifice and the anatomy, can influence the continued patency of the cyst. The cyst should be opened at or near the natural drainage point of the laryngeal saccule. Our technique entailed endoscopic marsupialization of the cyst without damaging the laryngeal framework. It is important to save the aryepiglottic fold which helps maintain the architecture of the supraglottic larynx and can possibly decrease the risk of aspiration.

Khodaei et al. [9] reported a newborn girl who developed marked inspiratory stridor and respiratory distress immediately after birth. During intubation by a senior anesthetist, a large laryngeal mass was noted in the supraglottic region. In our case, intubation was not possible until aspiration of the cyst.

Saccular cysts are rare and few reports on pediatric cases are found. While stridor in infants is not a rare entity, congenital laryngeal cysts are a rare but potentially fatal cause of airway obstruction within minutes after birth $[10,11]$. One case of a saccular cyst in a threemonth-old infant was described by Tosun et al. [12]. The infant presented with severe stridor and respiratory distress. Direct laryngoscopy of the larynx revealed a saccular cyst. The stridor disappeared shortly after surgical excision under direct laryngoscopy.

Another two cases of laryngeal cysts in adults, one a giant laryngocele that needed tracheostomy and the other, a large saccular cyst, were described by Pennings et al. [13]. Other case reports are found in small children and some authors favor complete excision by external cervical approach and others are for endoscopic removal of 
the cysts $[14,17,18]$.

Forensic medicine also described a 41-year-old man with a herniated saccular laryngeal cyst in the left cervical region who died unexpectedly at home from acute asphyxia [15]. The case is an example of a problem related to the possible evolution of laryngeal cysts, the mechanisms of asphyxial complications, the pathological diagnosis and the medico-forensic aspects. Another case of sudden death in a 36-year-old woman from a previously undiagnosed, asymptomatic laryngeal saccular cyst was described in Australia [16]. She presented with an acute, and consequentially fatal, airway obstruction. Difficulty during intubation, both in theater and in emergency settings, is a frequently presenting problem. This may have significant medico legal implications in determining possible negligence.

The prognosis of endoscopic removal of a saccular cyst depends on the specific case and the expertise of the surgical team. It is a very rare condition, but as seen in the literature, its prognosis is good. A few authors have described some recurrence after endoscopic procedures and prefer an open external approach. Our case was successful with the endoscopic approach and showed good prognosis with normal voice and swallowing function. Other studies presenting case series involving infants with large saccular cysts of the larynx and using the endoscopic procedure as a single intervention were also successful.

A literature search revealed only a few articles on congenital saccular cysts. To the best of our knowledge, airway obstruction due to saccular cyst during birth has not been previously published. The newborn described here with the difficult to perform intubation because of the cyst, makes this case unique. This case is a definite emergency that otolaryngologists, anesthesiologists and pediatric intensive care physicians should be aware of.

\section{Acknowledgements}

I. Braverman et al. thank Mrs. Ariela Ehrlich.

\section{REFERENCES}

[1] V. Forte, G. Fuoco and A. James, "A New Classification System for Congenital Laryngeal Cysts,” Laryngoscope, Vol. 114, No. 6, 2004, pp. 1123-1127. doi:10.1097/00005537-200406000-00031

[2] A. L. Abramson and B. Zielinski, "Congenital Saccular Cysts of the Newborn,” Laryngoscope, Vol. 94, No. 12, 1984, pp. 1580-1581. doi:10.1288/00005537-198412000-00009

[3] P. H. Holinger, L. D. Holinger, D. R. Barnes and L. J. Smid, "Laryngocele and Saccular Cysts," Annals of Otology, Vol. 87, No. 5, 1978, pp. 675-685.

[4] L. W. Desanto, K. D. Devine, L. H. Weiland, "Cysts of the Larynx, Classification,” Laryngoscope, Vol. 80, No. 1,
1970, pp. 261-267.

doi:10.1288/00005537-197001000-00013

[5] C. Arens, H. Glanz and O. Kleinsasser, "Clinical and Morphological Aspects of Laryngeal Cysts,” European Archives of Oto-Rhino-Laryngology, Vol. 254, No. 9-10, 1997, pp. 430-436. doi:10.1007/BF02439974

[6] S. M. Ahmad and A. M. Soliman, "Congenital Anomalies of the Larynx," Otolaryngologic Clinics of North America, Vol. 40, No. 1, 2007, pp. 177-191. doi:10.1016/j.otc.2006.10.004

[7] D. J. Kirse, C. J. Rees, A. W. Celmer and D. E. Bruegger, "Endoscopic Extended Ventriculotomy for Congenital Saccular Cysts of the Larynx in Infants," Archives of Otolaryngology-Head \& Neck Surgery, Vol. 132, No. 7, 2006, pp. 724-728. doi:10.1001/archotol.132.7.724

[8] C. A. Righini, H. Kadaoui, N. Morel, C. Llerena, E. Reyt, "Stridor in a Newborn Caused by a Congenital Laryngeal Saccular Cyst," International Journal of Pediatric Otorhinolaryngology Extra, Vol. 1, No. 2, 2006, pp. 145-149. doi:10.1016/j.pedex.2006.03.005

[9] I. Khodaei, A. Karkanevatos, A. Poulios and M. S. McCormick, "Airway Obstruction in a Newborn Due to a Congenital Laryngeal Cyst,” International Journal of Pediatric Otorhinolaryngology Extra, Vol. 2, No. 4, 2007, pp. 254-256. doi:10.1016/j.pedex.2007.08.001

[10] E. Ostfeld, Z. Hazan, S. Rabinson and L. Auslander, "Surgical Management of Congen Ital Supraglottic Lateral Saccular Cyst," International Journal of Pediatric Otorhinolaryngology, Vol. 19, No. 3, 1990, pp. 289-294. doi:10.1016/0165-5876(90)90010-O

[11] Z. Hazan, E. Ostfeld, S. Rabinson and L. Auslander, “Neonatal Respiratory Distress Due to Congenital Laryngeal Cyst,” Harefuah, Vol. 119, No. 11, 1990, pp. 371-372.

[12] F. Tosun, H. Söken and Y. Ozkaptan, "Saccular Cyst in an Infant, an Unusual Cause of Life-Threatening Stridor and Its Surgical Treatment," The Turkish Journal of Pediatrics, Vol. 48, No. 2, 2006, pp. 178-180.

[13] R. J. Pennings, F. J. van den Hoogen and H. A. Marres, "Giant Laryngoceles, a Cause of Upper Airway Obstruction,” European Archives of Oto-Rhino-Laryngology, Vol. 258, No. 3, 2001, pp. 137-140. doi:10.1007/s004050100316

[14] H. M. Danish, R. J. Meleca, J. P. Dworkin and T. R. Abbarah, "Laryngeal Obstructing Saccular Cysts, a Review of This Disease and Treatment Approach Emphasizing Complete Endoscopic Carbon Dioxide Laser Excision," Archives of Otolaryngology_Head \& Neck Surgery, Vol. 124, No. 5, 1998, pp. 593-596.

[15] E. Silingardi, N. Sola, A. L. Santunione and N. Trani, "Lateral Saccular Laryngeal Cyst and Unexpected Asphyxial Death,” Forensic Science International, Vol. 206, No. 1-3, 2011, pp. e17-e19.

[16] T. K. Kastowsky, M. P. Stevenson and J. A. Dufou, "Sudden Death from Saccular Laryngeal Cyst," Journal of Forensic Sciences, Vol. 51, No. 5, 2006, pp. 1144-1146. doi:10.1007/s004050100316

[17] V. Forte, J. Warshawski, P. Thorner and S. Conley, "Unusual Laryngeal Cysts in the Newborn,” International Journal of Pediatric Otorhinolaryngology, Vol. 37, No. 3, 
1996, pp. 261-267. doi:10.1016/0165-5876(96)01407-3

[18] M. H. Thabet and H. Kotob, "Lateral Saccular Cysts of the Larynx. Aetiology, Diagnosis and Management," The
Journal of Laryngology \& Otology, Vol. 115, No. 4, 2001, pp. 293-297. doi:10.1258/0022215011907488 